\title{
Perspective: Systems Pharmacology Strategy for Investigation of Antioxidant Drugs and Its Mechanisms of Action
}

\author{
Jianling Liu ${ }^{1}$, Chunli Zheng ${ }^{1}$ and Yonghua Wang ${ }^{2 *}$ \\ ${ }^{1}$ College of Life Science, Northwest University, Xi'an, Shaanxi, China \\ ${ }^{2}$ Center of Bioinformatics, College of Life Science, Northwest A \& F University, Yang ling, Shaanxi, China
}

Western medicine depends on science to create and evaluate drugs at the molecular level. In Asia, there is a commonly held belief that there is an art to healing too, and that both art and science should cooperate to help eradicate illness and relieve suffering [1]. The term systems pharmacology now describes a field of study that combines with reductionism (West) and holism (East) to develop an understanding of drug actions across multiple scales rooted in molecular interactions between the drug and its targets in the context of such targets interacting with and regulating other cellular components. This paper aims at understanding the antioxidation of herbal medicines from systems pharmacology point of view in the following three layers.

\section{How to Screen Antioxidant Molecules?}

Herbal medicine is a multifaceted system consisting of manifold components [2]. However, only a few of them act as active ingredients. Obtaining valid entities become a crucial step in drug discovery and herb mechanism revelation. Approaches to screen effective molecules in herbs count on experimental or in silico tools.

The traditional separation, purification and structure elucidation way is labor intensive, time-consuming and prone to error [3]. Owing to these reasons, in silico approaches appear to be a good alternative to the prediction of active components, such as ADME (absorption, distribution, metabolism, and excretion) evaluation. Various studies have developed their own models of ADME and, consequently, practices in drug molecular discovery [4-6]. Current ADME models commonly used mainly consist of human oral bioavailability $(\mathrm{OB})$ prediction model [7], drug-likeness (DL) evaluation method [8], blood brain barrier (BBB) [9], Caco2 [10], half-life (HL) prediction, and so forth. For example, to achieve more promising drugs, Huang, et al. defined the filtering criteria as $\mathrm{DL} \geq 0.18$; $\mathrm{OB} \geq 30 \%$; $\mathrm{BBB} \geq 0$ or Caco2 $\geq-0.4$ (2). With the aid of in silico models, a global number of compounds are boiled down to better suit subsequent analysis.

In particular, with the continuous advance of -omics technologies, pharmaceutical chemists are interested in hybrid molecules consisting of two distinct drug entities covalently linked in a single molecule [11]. The two distinct drug entities were purified from plant cell culture or chemical synthesis.

\section{How to Detect Antioxidant Targets?}

Target discovery, which involves the identification and early validation of disease modifying targets, is an essential first step in the drug discovery pipeline [12]. The 'front-loading' of this topic makes for tackling the issues related to failures of on-target biological hypotheses and on- and off-target safety concerns. Indeed, the drive to determine antioxidation targets has made substantial progress, both in industry and academia, with the ever-increasing number of novel and innovative technologies for target discovery. We critically examine the front methodologies used for both the identification and validation of disease-relevant proteins and provide a comparative review in the three broad target discovery strategies: genetic interaction and genomic methods; computational inference methods; and high-throughput screening.

\section{Genetic interaction methods}

Genetic interaction is an interaction between multiple genes that impacts the expression of the phenotype (positive or negative). This method provides a productive avenue for expanding target sets and tackling core issues in drug development. Global genetic networks would identify numerous pairs of nonessential genes that can be targeted in combination to reveal pharmacological effects, and thus the target space is expanded well beyond that of the subset of essential genes. In addition, interactions with conditional alleles of essential genes also enable the identification of genes and pathways that buffer the activity of an essential cellular function and thereby predict targets whose cognate inhibitors might show strong synergistic activity with the existing drugs [13]. As illustrated above, the genetic interaction strategy provide a landscape to rationally expand the existing target space address the issue of target identification in early stages of drug discovery.

\section{Computational inference methods}

Computational inference methods have been introduced to predict true interacting drug-target pairs with high accuracy. These methods are motivated by the observation that similar drugs tend to target similar proteins $[14,15]$. A desirable property of these methods is that it does not require the $3 \mathrm{D}$ structure information of the target proteins, which is needed in traditional methods based on docking simulations [16]. This property, for instance, chemical similarity [17], has already successfully applied to the prediction of drug-target interaction for herbal medicines [18,19]. Comparing with the nonin silico approaches, computational inference methods make a huge amount of data accessible and processable, which can help not only to avoid animal testing but also for screening purposes and significantly decrease drug development costs [20].

\section{High-throughput screening}

High-throughput screening (HTS) has become an important part of drug discovery at most pharmaceutical and many biotechnology companies worldwide, and use of HTS technologies is expanding into

*Corresponding author: Yonghua Wang, Center of Bioinformatics, College of Life Science, Northwest A \& F University, Yang ling, Shaanxi, China, Tel: +86-02987092262; E-mail: yh_wang@nwsuaf.edu.cn

Received March 17, 2014; Accepted March 19, 2014; Published March 27, 2014

Citation: Liu J, Zheng C, Wang Y (2014) Perspective: Systems Pharmacology Strategy for Investigation of Antioxidant Drugs and Its Mechanisms of Action. J Bioequiv Availab 6: e50. doi:10.4172/jbb.10000e50

Copyright: @ 2014 Liu J, et al. This is an open-access article distributed under the terms of the Creative Commons Attribution License, which permits unrestricted use, distribution, and reproduction in any medium, provided the original author and source are credited. 
target validation [21]. The advent of powerful genomics technologies has uncovered many fundamental aspects of biology. Particularly, chemical genomics, which seeks to identify the functional relationship between specific genes and compounds via a systematic analysis of all genes in a genome, allows the genomewide identification of potential drug targets [22]. In summary, HTS is a powerful technology that holds great promise for advancing pharmacologically relevant targets.

\section{How to Bridge Antioxidant and Diseases?}

The tremendous amount of the data extracted from the study of complex biological systems changes our view on the pathogenesis of human diseases. Instead of staring at individual components of biological processes, systems pharmacology focuses our attention more on the interaction and dynamics of biological systems. A network representation and analysis of the physiology and pathophysiology of biological systems is an effective way to study their complex behavior [23]. Understanding diseases in the context of network principles allows us to address some fundamental properties of the antioxidant that are involved in disease. In the following, we briefly discuss the most studied network maps and their limitations, but we warn the reader to exercise caution as we are describing a promptly altering domain.

\section{Molecular-level network}

A network is defined as a series of entities connected to one another on the basis of a defined criterion [24]. The entities in a network are named nodes, which represent different types of molecules such as DNA, RNA, targets, and drugs. In the context of studying relationships of antioxidant and diseases, edges between nodes may represent DNARNA or RNA-RNA interactions [25], protein-protein interactions [26], drug-target interactions [27], target-target interactions [28], and drugdrug interactions [29]. Typically, both the nodes and the interactions discussed above need to be evaluated in the context of tissue specificity, as they may exert a functional role on the context of only selected tissues [30]. Still, human interactome maps remain incomplete and noisy, a fact that needs to be taken into account when using them to study diseases and systematic efforts should be paid to increase the coverage of human interactome maps.

\section{Cellular/ tissue-level network}

As evolution evolved more complex multicellular organisms and biological behaviors, cells are capable of interacting with one another through diverse biological and physical mechanisms. A depiction of cell-cell network is absolutely necessary for an understanding of how cellular activity is coordinated in information exchange and functional entities (such as tissues) recognition. Describing such systems has always been restricted by the barrier of experimenting on living tissues. And computer modelling appears actually compelling to characterize the performance of multicellular systems. A generic model dedicated to the analysis and modelling of plant morphogenesis was constructed by describing the plant as a multi-scale structure [30]. However, modelling studies remain have not been integrated into life-like systems, there is still a great potential to develop in terms of computer modelling.

\section{Organ-level network}

One important goal in systems pharmacology is to develop molecular diagnostics that can accurately and comprehensively report health and disease states of an organ system [31]. To address this issue, a number of sophisticated networks of organ-level were built. On the basis of the fact that some therapeutic targets are highly and selectively expressed in specific tissues, network, to announce the relationship of target location and disease, among heart, brain, liver and so forth was constructed $[32,33]$. On the other hand, the discovery of organlevel molecular signatures from global biomolecule expression measurements would mark a significant advance toward this goal [34]. In summary, developing networks at various scales allows us to explicitly track drug effects from molecule level interactions to organismal physiology.

\section{Acknowledgements}

The research is supported by the Fund of Northwest A \& F University and is financially supported by the National Natural Science Foundation of China (Grant No. 31170796 and 81373892)

\section{References}

1. Liu L, Leung EL, Tian X (2011) Perspective: The clinical trial barriers. Nature 480: S100.

2. Huang C, Zheng C, Li Y, Wang Y, Lu A, et al. (2013) Systems pharmacology in drug discovery and therapeutic insight for herbal medicines. Brief Bioinform.

3. Tao W, Xu X, Wang X, Li B, Wang Y, et al. (2013) Network pharmacologybased prediction of the active ingredients and potential targets of Chinese herbal Radix Curcumae formula for application to cardiovascular disease. J Ethnopharmacol 145: 1-10

4. Li X, Xu X, Wang J, Yu H, Wang X, et al. (2012) A system-level investigation into the mechanisms of Chinese Traditional Medicine: Compound Danshen Formula for cardiovascular disease treatment. PLoS One 7: e43918.

5. Wang X, Xu X, Li Y, Li X, Tao W, et al. (2013) Systems pharmacology uncovers Janus functions of botanical drugs: activation of host defense system and inhibition of influenza virus replication. Integrative Biology 5: 351-71.

6. Wang X, Xu X, Tao W, Li Y, Wang Y, et al. (2012) A systems biology approach to uncovering pharmacological synergy in herbal medicines with applications to cardiovascular disease. Evid Based Complement Alternat Med 2012: 519031.

7. Xu X, Zhang W, Huang C, Li Y, Yu H, et al. (2012) A novel chemometric method for the prediction of human oral bioavailability. Int J Mol Sci 13: 6964-6982.

8. Liu H, Wang J, Zhou W, Wang Y, Yang L (2013) Systems approaches and polypharmacology for drug discovery from herbal medicines: an example using licorice. J Ethnopharmacol 146: 773-793.

9. Li L-t, Li Y, Wang Y-h, ZHANG SW (2007) Prediction of BBB permeation based on molecular indices. Chinese Journal of Medicinal Chemistry 17: 221

10. Wessel MD, Jurs PC, Tolan JW, Muskal SM (1998) Prediction of human intestinal absorption of drug compounds from molecular structure. J Chem Inf Comput Sci 38: 726-735.

11. (Chinese) Saompc-n.

12. Lindsay MA (2003) Target discovery. Nat Rev Drug Discov 2: 831-838.

13. Roemer T, Boone C (2013) Systems-level antimicrobial drug and drug synergy discovery. Nat Chem Biol 9: 222-231.

14. Klabunde $T$ (2007) Chemogenomic approaches to drug discovery: similar receptors bind similar ligands. Br J Pharmacol 152: 5-7.

15. Schuffenhauer A, Floersheim P, Acklin P, Jacoby E (2003) Similarity metrics for ligands reflecting the similarity of the target proteins. J Chem Inf Comput Sci 43: 391-405

16. Cheng AC, Coleman RG, Smyth KT, Cao Q, Soulard P, et al. (2007) Structurebased maximal affinity model predicts small-molecule druggability. Nat Biotechnol 25: 71-75.

17. Yu H, Chen J, Xu X, Li Y, Zhao H, et al. (2012) A systematic prediction of multiple drug-target interactions from chemical, genomic, and pharmacological data. PLoS One 7: e37608.

18. Yao Y, Zhang X, Wang Z, Zheng C, Li P, et al. (2013) Deciphering the combination principles of Traditional Chinese Medicine from a systems pharmacology perspective based on Ma-huang Decoction. Journal of Ethnopharmacology 150: 619-638.

19. Li P, Chen J, Wang J, Zhou W, Wang X, et al. (2013) Systems pharmacology strategies for drug discovery and combination with applications to CVD. Journal of Ethnopharmacology 152: 93-107. 
Citation: Liu J, Zheng C, Wang Y (2014) Perspective: Systems Pharmacology Strategy for Investigation of Antioxidant Drugs and Its Mechanisms of Action. J Bioequiv Availab 6: e50. doi:10.4172/jbb.10000e50

Page 3 of 3

20. Haupt VJ, Schroeder M (2011) Old friends in new guise: repositioning of known drugs with structural bioinformatics. Brief Bioinform 12: 312-326.

21. Fox S, Farr-Jones S, Sopchak L, Boggs A, Nicely HW, et al. (2006) Highthroughput screening: update on practices and success. J Biomol Screen 11: 864-869

22. Sun Z, Sun Y, Zhou Y, Wan Y (2012) Yeast Genomics Technique for HighThroughput Drug Target Discovery. Drug Development Research 73: 398-405.

23. del Sol A, Balling R, Hood L, Galas D (2010) Diseases as network perturbations. Curr Opin Biotechnol 21: 566-571.

24. Zhao S, lyengar R (2012) Systems pharmacology: network analysis to identify multiscale mechanisms of drug action. Annual Review of Pharmacology and Toxicology 52: 505-521.

25. Sumazin P, Yang X, Chiu HS, Chung WJ, lyer A, et al. (2011) An extensive microRNA-mediated network of RNA-RNA interactions regulates established oncogenic pathways in glioblastoma. Cell 147: 370-381.

26. Schneider CM, de Arcangelis L, Herrmann HJ (2011) Modeling the topology of protein interaction networks. Phys Rev E Stat Nonlin Soft Matter Phys 84 016112.
27. Yildirim MA, Goh KI, Cusick ME, Barabási AL, Vidal M (2007) Drug-target network. Nat Biotechnol 25: 1119-1126.

28. Xu Y, Guo M, Liu X, Wang C, Liu Y (2014) Inferring the soybean (Glycine max) microRNA functional network based on target gene network. Bioinformatics 30 94-103.

29. Hu G, Agarwal P (2009) Human disease-drug network based on genomic expression profiles. PLoS One 4: e6536.

30. Barabási AL, Gulbahce N, Loscalzo J (2011) Network medicine: a networkbased approach to human disease. Nat Rev Genet 12: 56-68.

31. Tian Q, Price ND, Hood L (2012) Systems cancer medicine: towards realization of predictive, preventive, personalized and participatory (P4) medicine. J Intern Med 271: 111-121.

32. Zheng CJ, Han LY, Yap CW, Ji ZL, Cao ZW, et al. (2006) Therapeutic targets: progress of their exploration and investigation of their characteristics. Pharmacol Rev 58: 259-279.

33. Blagosklonny MV (2003) Tissue-selective therapy of cancer. Br J Cancer 89 1147-1151.

34. Sung J, Kim PJ, Ma S, Funk CC, Magis AT, et al. (2013) Multi-study integration of brain cancer transcriptomes reveals organ-level molecular signatures. PLoS Comput Biol 9: e1003148. 